\title{
Models of Reduction ${ }^{1}$
}

\author{
OtÁvio Bueno \\ University of Miami
}

\begin{abstract}
In this paper, I examine three models of reduction. The first, and the most restrictive, is the model developed by Ernest Nagel as part of the logical empiricist program. The second, articulated by Jerry Fodor, is significantly broader, but it seems unable to make sense of a salient feature of scientific practice. The third, and the most lenient, model is developed within Newton da Costa and Steven French's partial structures approach. I argue that the third model preserves the benefits of Fodor's proposal, and it is still able to accommodate relevant aspects of scientific practice. In particular, it offers a conception of reduction without reductionism, and an account of the relation between reduced and reducing theories via unsharp mappings and partial structures-even in the presence of incomplete information.
\end{abstract}

Keywords: Reduction, models, special sciences, partial structures, partial mappings.

\section{Introduction}

The concept of reduction has played a significant role in early conceptions of science, particularly those articulated by logical empiricists, such as Rudolf Carnap (1928/1967) and Ernest Nagel (1961). It also played an important role in philosophical reconstructions of mathematics in the hands of Gottlob Frege $(1893,1903)$, with the reduction of arithmetic to second-order logic plus definitions. Part of the difficulty of these early approaches to reduction is the extremely tight connection they require between the domains under study: the reducing and the reduced.

In response to this sort of concern, Jerry Fodor has offered a very interesting, and much less restrictive, model of reduction, with the additional bonus of preserving the autonomy of the special sciences (see Fodor 1974 and 1997). This is clearly the right move to make. But Fodor's proposal faces a difficulty to accommodate certain aspects of scientific practice, in particular given the use of quantum mechanics in chemistry. In this paper, building on Fodor's approach, I offer an alternative, also less restrictive, model of reduction. In order to do that, I explore the resources of Newton da Costa and Steven French's partial structures approach (see da Costa and French 2003), and examine in which way we can still consider a place for reduction in contemporary science. 


\section{Two models of reduction}

To set out the scene, I will start by considering two models of reduction: Nagel's reduction and Fodor's reduction. ${ }^{2}$ Nagel's reduction is obtained via derivability: one is supposed to establish a derivation relation between reduced and reducing theories. The proposal is inspired in Frege's approach to the logical foundations of arithmetic, and it extends the approach to the empirical sciences. Similarly to Frege's, Nagel's proposal demands a very strict relation between the theories involved. In contrast, Fodor's reduction is obtained via the introduction of disjunctive properties. The proposal offers a significantly less restrictive connection between the reducing and reduced theories. And, in particular, it allows for exceptions in the laws of the special sciences-a much more promising strategy. I will critically examine each of these models of reduction in turn.

\subsection{Nagel's reduction}

Nagel conceptualizes the reduction between two theories in terms of one theory explaining the other, where the model of explanation that he adopts is the deductivenomological account. He emphasizes the point:

Reduction $[\ldots]$ is the explanation of a theory or a set of experimental laws established in one area of inquiry, by a theory usually though not invariably formulated for some other domain. (Nagel 1961: 338.)

Given the account of explanation that is used-according to which an explanation is ultimately a deductively valid argument - the relation between the reducing and the reduced theory becomes one of derivation. To reduce a theory $T$ to a theory $T^{\prime}$, we should be able to derive the principles or laws that characterize $T$ from those of $T^{\prime}$.

However, Nagel identifies a difficulty that emerges immediately given the requirement of logical derivability:

If the laws of the secondary science [that is, the reduced theory] contain terms that do not occur in the theoretical assumptions of the primary discipline [that is, the reducing theory] [...] the logical derivation of the former from the latter is prima facie impossible. (Nagel 1961: 352.)

Clearly, if the reduced and reducing theories don't have a shared vocabulary, no logical derivation between them could be established.

In order to address this difficulty, Nagel introduces two "necessary formal conditions" that need to be in place so that the reduction can occur. (a) The first is the existence of bridge laws, which are used to link the vocabularies of the reduced and the reducing theories. 
Assumptions of some kind must be introduced which postulate suitable relations between whatever is signified by ' $\mathrm{A}$ ' [i.e. the offending term in the reduced theory] and traits represented by theoretical terms already present in the primary [reducing] science. (Nagel 1961: 353.)

The assumptions Nagel refers to above are precisely the bridge laws. (b) The second required formal condition is derivability. In order to be able to reduce one theory to another, on Nagel's model, one needs to be able to derive the laws of the reduced theory from those of the reducing one. As Nagel points out:

With the help of these additional assumptions (the bridge laws], all the laws of the secondary science, including those containing the term ' $\mathrm{A}$ ', must be logically derivable from the theoretical premises and their associated coordinating definitions in the primary discipline. (Nagel 1961: 353-4.)

If no such derivability can be established between the theories in question, there is no reduction between them.

Given the crucial role played by bridge laws in Nagel's reduction, various issues surface (see Batterman 2002: 63). What exactly is the status of the bridge laws? That is, how should these laws be understood? Do they emerge from some sort of linguistic analysis? Or are they empirical discoveries? If bridge laws are empirical statements, what kind of necessity do they display? In particular, if bridge laws establish identity relations among suitable expressions of the reducing and the reduced theories, are such identity relations synthetic or analytic? The development of Nagel's conception presupposes answers to all of these questions.

Let me focus on the last question. It seems clear that, in order for Nagel's approach to work, the bridge laws need to establish some kind of synthetic identity relation (Batterman 2002: 63). Consider the reduction of the theory of physical optics to that of electromagnetic radiation. The reduction is implemented by identifying two groups of entities: light waves and electromagnetic radiation. As Lawrence Sklar points out:

The place of correlatory laws [i.e. bridge laws] is taken by empirically established identifications of two classes of entities. Light waves are not correlated with electromagnetic waves, for they are electromagnetic waves. (Sklar 1967: 120.)

It is, therefore, crucial to establish, empirically, the identity of the relevant objects. What Nagel needs, before Saul Kripke, are necessary a posteriori identity statements.

It turns out, however, that Nagel's proposal faces several difficulties. (1) Strictly speaking, we hardly ever have logical derivations in scientific practice. Thus, we hardly ever find actual cases of reduction in Nagel's sense. The paradigmatic example that Nagel offers of reduction is the reduction of the Galilean laws of falling

Principia 13(3): 269-82 (2009). 
bodies to Newtonian mechanics. But not even this case actually works. At best, we can derive from Newtonian mechanics approximations to the laws of the Galilean theory. But these approximations are, in fact, incompatible with the actual laws. Thus, no logical derivations are actually established between the two theories (see Sklar 1967: 110-1, and Batterman 2002: 62).

(2) Even if we could derive the reduced theory from the reducing one, the latter typically corrects the former (Feyerabend 1962). Consider, for instance, the concept of temperature from classical thermodynamics. This is a nonstatistical concept, characterized in terms of Carnot cycles, and it is related to the nonstatistical second law of thermodynamics. Consider, now, the alleged reduction of classical thermodynamics to statistical mechanics. The "reduction" doesn't identify nonstatistical items in the reducing theory (statistical mechanics) with the nonstatistical concept of temperature as the latter is found in the reduced theory (thermodynamics). In fact, given that, as opposed to statistical mechanics, thermodynamics is not a statistical theory, it is unclear how we could even find a bridge law that captures the concept of temperature and the function it plays in thermodynamics. That is, it is unclear how the alleged reduction could ever be established (see Batterman 2002: 64).

Perhaps the Nagelian could take the point. She could incorporate into the characterization of reduction the idea that the reduced theory ends up being corrected by the reducing theory. That is, the outcome of the reduction process may well be a corrected version of the reduced theory. In fact, several physicists now would agree that the concept of temperature in classical thermodynamics and other concepts in that theory, such as entropy, have to be changed in light of the "reduction" to statistical mechanics. Physicists even speak of the theory of statistical thermodynamics. Thus, reduction can play an important role in the process of correction and refinement of scientific theories.

Schaffner (1976) explicitly incorporates into a Nagelian framework the idea of a "corrected reduced theory" (Shaffner 1976: 618). A theory $T$ reduces a theory $T^{\prime}$ if, and only if, there is a corrected version of $T^{\prime}$, call it $T^{*}$, such that:

(a) the primitive terms of $T^{* *}$ are associated via bridge laws with various terms of $T$;

(b) $T^{*}$ is derivable from $T$ when it is supplemented with such bridge laws;

(c) $T^{*}$ corrects $T^{\prime}$ in that it makes more accurate predictions than $T^{\prime}$ does;

(d) $T^{\prime}$ is explained by $T$ in that $T^{\prime}$ and $T^{\prime *}$ are strongly analogous to one another, and $T$ indicates why $T^{\prime}$ works as well as it does in its domain of validity.

Schaffner's proposal is very reasonable, given the Nagelian framework it assumes. But perhaps rather than theory reduction, what Schaffner offers is an ac- 
count of a different concept: what we are dealing with here is a process of theory refinement (see also Batterman 2002: 64-5).

(3) Even the revised Nagelian approach does not quite work due to the phenomenon of multiple realization (Fodor 1974 and 1997; see Batterman 2002: 6568). The central idea is that "upper level" properties (such as psychological states) are potentially realized in a huge variety of heterogeneous lower level physical states. This multiple realizability would make it impossible to establish the bridge laws that, on the Nagelian approach, are required for the derivation of the laws of the reduced theory (in the special sciences) from the laws of the reducing theory (e.g. physics). The central point is the heterogeneity of the lower level realizers of the properties of the upper level special sciences. The heterogeneity makes it impossible to identify the relevant items in the bridge laws. (Recall that the latter requires a necessary $a$ posteriori identity relation.)

Given these difficulties with the Nagelian approach to reduction, a different, more lenient, model is in order. Fodor offers this model.

\subsection{Fodor's reduction}

Fodor's approach to reduction is shaped, in crucial ways, by the multiple realizability problem just mentioned. In his own words, here is how he presents the problem:

The very existence of the special sciences testifies to reliable macrolevel regularities that are realized by mechanisms whose physical substance is quite typically heterogeneous. [...] Damn near everything we know about the world suggests that unimaginably complicated to-ings and fro-ings of bits and pieces at the extreme microlevel manage somehow to converge on stable macrolevel properties. On the other hand, the 'somehow' really is entirely mysterious $[\ldots]$. [Some do not] see why there should be (how there could be) [macrolevel regularities] unless, at a minimum, macrolevel kinds are homogeneous in respect of their microlevel constitution. Which, however, functionalists in psychology, biology, geology, and elsewhere, keep claiming that they typically aren't. (Fodor 1997: 160-1.)

Given the multiple realizability phenomenon, it comes as no surprise that Fodor is a not a (classical) reductionist, and certainly not of a Nagelian sort. Instead, he offers an alternative, less stringent account of the relation of the special sciences to physics, and defends a corresponding weaker claim: token physicalism (Fodor 1974). But what exactly is token physicalism? As he notes:

If the bridge laws express event identities, and if every event that falls under the proper laws of a special science falls under a bridge law, we get the truth of a doctrine that I shall call 'token physicalism'. Token physicalism is simply the claim that all the events that the sciences talk about are physical events. (Fodor 1974: 100.)

Principia 13(3): 269-82 (2009). 
In other words, the crucial feature of token physicalism is the idea that, ultimately, the events described by the sciences are physical.

It is important, however, to distinguish token physicalism from type physicalism. According to type physicalism, "every property mentioned in the laws of any science is a physical property" (Fodor 1974: 100). Token physicalism is weaker than type physicalism, given that the former does not entail, but is entailed by, the latter. After all, "the contingent identity of a pair of events presumably does not guarantee the identity of the properties whose instantiation constitutes the events; not even when the event identity is nomologically necessary" (Fodor 1974: 100). Type physicalism, though, does entail token physicalism. For "if an event is simply the instantiation of a property [...], two events will be identical when they consist of the instantiation of the same property by the same individual at the same time" (Fodor 1974: 100). Fodor is only committed to the weaker, and more plausible, form of physicalism.

What is the connection between token physicalism and reductionism (or reductivism)? Here is Fodor's answer:

Reductivism [or reductionism] is the conjunction of token physicalism with the assumption that there are natural kind predicates in an ideally completed physics which correspond to each natural kind predicate in any ideally completed special science. (Fodor 1974: 100.)

Note the sudden introduction of (i) natural kinds, and (ii) the requirement that there be a correspondence between natural kind predicates in a completed physics with each natural kind predicate in the completed special sciences. But what exactly would count as an "ideally completed physics" and an "ideally completed special science"? Can we really make sense of what such a science would be like? Neither natural kinds nor the idea of an "ideally completed science" are found in Nagel's conception of reduction.

Given this conception of reductionism, it is not surprising that Fodor wants to move away from it. As he emphasizes:

It will be one of my morals that the truth of reductivism [reductionism] cannot be inferred from the assumption that token physicalism is true. Reductivism is a sufficient, but not a necessary, condition for token physicalism. (Fodor 1974: 100-1.)

Instead of a strict reductionist picture, Fodor offers a different model of reduction altogether. The diagram below illustrates the central features of Fodor's reduction (see Fodor 1974: 109).

Principia 13(3): 269-82 (2009). 
(a) Law of special science:

(b) Bridge statements:

(c) Disjunctive predicates of reducing science:

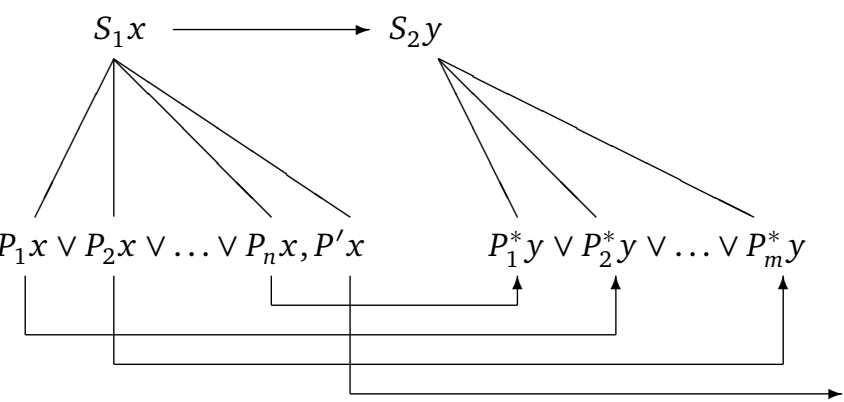

(d) Laws of reducing science

Figure 1: Fodor's reduction

Fodor's proposal has, in outline, four central aspects:

(i) The proposal allows us to see how the laws of the special sciences can reasonably have exceptions-a well-known fact about these sciences. Any $S_{1}$ event of the type $P^{\prime}$-for which there is no corresponding $S_{2}$ event-will be an exception to the law $S_{1} x \rightarrow S_{2} x$.

(ii) Fodor's proposal does not require that bridge statements be laws. Given the multiple realizability phenomenon bridge statements will inevitably connect disjunctive predicates. Fodor doubts that disjunctive predicates can be taken to be kind predicates (Fodor 1974: 109), but given that he also takes laws to be connections among natural kinds (Fodor 1974: 101-2), it follows that, on his view, bridge statements are not laws.

(iii) Fodor's proposal is weaker than reductionism (in Fodor's sense). After all, the proposal "does not imply a correspondence between the kind predicates of the reduced and the reducing science" (Fodor 1974: 110).

(iv) Finally, Fodor's proposal implies physicalism. After all, his proposal satisfies "the same assumption that makes standard reductivism [reductionism] physicalistic (namely, that bridge statements express true token identities)" (Fodor 1974: 110).

Although in many ways Fodor's proposal offers an interesting alternative to Nagel's account, it is unclear that the proposal can make sense of a central feature of scientific practice. To illustrate this point, I will discuss an example that explores the connection between chemistry and quantum mechanics, and which raises a difficulty for Fodor's proposal. I will then indicate a way of accommodating the difficulty, by offering a third model of reduction via unsharp mappings and partial structures, which still preserves the central features of Fodor's account. 


\section{A third model of reduction: unsharp and partial mappings}

In order to motivate the third model of reduction, I will consider an aspect of the relation between chemistry and non-relativist quantum mechanics (QM) as it emerges in the context of actual chemical practice. One of the most unexpected features of $\mathrm{QM}$ is the way in which, at least on a significant interpretation of the theory, the concept of identity cannot be applied to elementary particles. ${ }^{3}$ According to Erwin Schrödinger, one of the main proponents of this view:

As our mental eye penetrates into smaller and smaller distances and shorter and shorter times, we find nature behaving so entirely differently from what we observe in visible and palpable bodies of our surroundings that no model shaped after our large-scale experiences can ever be true. (Schrödinger 1952: 17-8.)

In particular, as Schrödinger insists (1952: 17-8), one of the most significant differences between the macroscopic and the microscopic worlds is that the concept of identity lacks any sense if it is applied to elementary particles.

Now, if quantum particles lack identity conditions, it is unclear how exactly the laws of quantum mechanics can be used to explain how "larger" objects that are studied in chemistry (such as molecules, inorganic crystals etc.) do have identity conditions. Moreover, if quantum particles lack identity conditions, it is unclear how we will be able to express a bridge statement that connects chemical predicates (such as, " $x$ is a crystalline inorganic solid") with corresponding quantum mechanical predicates involving quantum particles (such as " $x$ is the electron that..."). After all, on Schrödinger's interpretation, "the electron" is not a meaningful expression. And if such bridge statements are not available, Fodor's proposal cannot get off the ground, given that, ultimately, it depends on the identification of items in the reducing science to items in the special science. Given the presumed lack of identity conditions for elementary particles, how do chemists manage to do their own work?

As is well known, we can divide crystalline inorganic solids into three groups: metals, semiconductors, and insulators. All of them have bands, that is, nearly continuous energy levels. The central different is that while metals have a partially filled band, semiconductors have a completely filled band (called the "valence band"), which is separated from the (mostly empty) conduction band by a bandgap $E_{g}$. Insulators, in turn, have the same electronic structure as semiconductors, except that they have a larger bandgap $E_{g}$. As a result, insulators are poor conductors, since they demand more energy to shift electrons from one band to another. Quantum dots are semiconductors with all three dimensions in the 1-10 nanometer $(\mathrm{nm})$ size range (see Murphy and Coffer 2002).

Let us now suppose that a semiconductor is irradiated with light of energy $h v>$ $E_{g}$. In this case, Murphy and Coffer note:

Principia 13(3): 269-82 (2009). 
An electron will be promoted from the valence band to the conduction band, leaving a positively charged "hole" behind. This hole can be thought of as the absence of an electron and acts as a particle with its own effective mass and charge in the solid. (Murphy and Coffer 2002: 17A.)

And suppose that we are interested in calculating the spatial separation of the electron and its hole (which is called an "exciton"). We can carry out the calculation by invoking a modified Bohr model. ${ }^{4}$ As Murphy and Coffer indicate:

For typical semiconductor dielectric constants, the calculation suggests that the electron-hole pair spatial separation is $\sim 1-10 \mathrm{~nm}$ for most semiconductors. In this size range, when the exciton is created, the physical dimensions of the particle confine the exciton in a manner similar to the particle-in-abox problem of physical chemistry. Therefore, the quantum effects such as quantization of energy levels can be observed in principle. (Murphy and Coffer 2002: 17A.)

If we note the way in which Murphy and Coffer refer to electrons in this context, it becomes clear what is needed in order to refer to electrons despite their assumed lack of identity conditions. What we need is a broader notion of mapping: an unsharp mapping from the relevant models of the reducing science (QM) to the relevant models of the special science (chemistry), which does not require that the things that are being mapped have well-defined identity conditions. The mapping is unsharp in that instead of being defined for each individual element of its domain, it is defined for equivalence classes of elements of the domain (see Bueno [2006]). With such an unsharp mapping in place, we can make sense of the way in which chemists can do their work even if they are dealing with elementary particles that lack identity conditions.

Note, once again, the way in which Murphy and Coffer describe the situation:

An electron will be promoted from the valence band to the conduction band $[\ldots]$. This hole can be thought of as the absence of an electron and acts as a particle [...]. (Murphy and Coffer 2002: 17A; italics added.)

Clearly, identity conditions for electrons are never asserted here, nor is it even presupposed that electrons have identity conditions. All that is required is that we pick out an electron from a whole bunch of indistinguishable electrons-any one of them will do. Instead of a precise way of uniquely picking out a particular electron, all we need is an unsharp mapping from equivalence classes of electrons to the Bohr model, and from that model to the models that describe the chemical phenomena under consideration. In other words, what we need are unsharp mappings between the models of the reducing theory $(\mathrm{QM})$ and the models of the special science (chemistry).

Principia 13(3): 269-82 (2009). 
Suitably interpreted in terms of unsharp mappings, Fodor's broader model of reduction offers an interesting account of the relation between the special sciences and the reducing science. But we need not assume the metaphysics of natural kinds and laws of nature to appropriate the structure of Fodor's model. In fact, those who favor an empiricist outlook — and I am certainly among them-are likely to remain agnostic about the existence of such items (see van Fraassen [1989]). So just the overall structure of Fodor?s model needs to be adopted to formulate the unsharp mappings approach. The central move is to introduce unsharp mappings to link the models of the reducing and the reduced theories-particularly in those cases in which the reducing science involve elementary particles, and hence we face the difficulty of being unable to identify the events in question, given the presumed lack of well-defined identity conditions for such particles.

Clearly, there are cases in which the special science and the reducing science do not depend directly on QM, and hence the unsharp mappings need not be invoked. (The latter will be used, though, when we get to quantum mechanical descriptions.) However, even in the cases that do not depend on QM, we will need to accommodate the ubiquitous fact that we do not have complete information about the domains under consideration, including those described by our best theories (see da Costa and French [2003]). In this case, in order to develop a concept of "reduction" that is sensitive to the informational limitations that characterize scientific practice, we need to offer connections between the models of the reducing and the reduced theories that take into account what is still unknown about the relevant domains. Partial structures (structures that represent the partiality of information about such domains) and partial mappings (transformations that connect partial structures, preserving the partiality of information) can then be introduced (see da Costa and French [2003]). These weaker mappings between partial structures involve transformations, such as partial isomorphism and partial homomorphism, which can be applied even in contexts of partial information (see Bueno, French, and Ladyman [2002]).

But what, exactly, is a partial structure ${ }^{5}$ A partial structure is an ordered pair $\left\langle\mathrm{D}, R_{i}\right\rangle_{i \in I}$, where $D$ is a non-empty set, and $\left(R_{i}\right)_{i \in I}$ is a family of partial relations defined over $D$. In turn, an $n$-place partial relation $R$ over $D$ is a triple $\left\langle R_{1}, R_{2}, R_{3}\right\rangle$, where $R_{1}, R_{2}$, and $R_{3}$ are mutually disjoint sets, with $R_{1} \cup R_{2} \cup R_{3}=D^{n}$, and such that: $R_{1}$ is the set of $n$-tuples that (we know that) belong to $R ; R_{2}$ is the set of $n$-tuples that (we know that) do not belong to $R$, and $R_{3}$ is the set of $n$-tuples for which it is not known (or, for reasons of simplification, it is ignored that it is known) whether they belong or not to $R$. (Notice that if $R_{3}$ is empty, $R$ is a usual $n$-place relation that can be identified with $R_{1}$.)

As noted, partial structures and partial relations offer a way of representing the partiality of information about a given domain of inquiry. As an illustration, suppose that this domain is the physics of particles, and let us denote it by $\Delta$. In order to 
study this domain, researchers formulate a conceptual framework that helps them systematize and interpret the information they obtain about $\Delta$. This domain can be represented by a set $D$ of objects (which includes real objects, such as configurations in a Wilson chamber and spectral lines, and "ideal" objects, such as quarks). $D$ is studied by the examination of the relations that hold among its elements. However, it often happens that, given a relation $R$ defined over $D$, we just do not know whether $R$ relates all objects of $D$ (or $n$-tuples thereof). Moreover, we often need to ignore some of the relations that are known to hold among objects of $D$ in order to study other relations in that domain in a tractable way. This is part of the incompleteness and partiality of our information about $\Delta$, and is formally accommodated by partial relations and partial structures.

In terms of these structures, appropriate characterizations of partial isomorphism and partial homomorphism can then be offered (see Bueno, French and Ladyman, 2002). Given that the latter notions are more open-ended than the standard ones, they accommodate better the partiality of structures found in scientific practice. Let $S=\left\langle D, R_{i}\right\rangle_{i \in I}$ and $S^{\prime}=\left\langle D^{\prime}, R_{i \in I}^{\prime}\right.$ be partial structures. So, each $R_{i}$ is a partial relation of the form $\left\langle R_{1}, R_{2}, R_{3}\right\rangle$, and each $R_{i}^{\prime}$ a partial relation of the form $\left\langle R_{1}^{\prime}, R_{2}^{\prime}, R_{3}^{\prime}\right\rangle .^{6}$

We say that a partial function ${ }^{7} f: D \rightarrow D^{\prime}$ is a partial isomorphism between $S$ and $S^{\prime}$ if (i) $f$ is bijective, and (ii) for every $x$ and $y \in D, R_{1} x y \leftrightarrow R_{1}^{\prime} f(x) f(y)$ and $R_{2} x y \leftrightarrow R_{2}^{\prime} f(x) f(y)$. So, when $R_{3}$ and $R_{3}^{\prime}$ are empty (that is, when we are considering total structures), we have the standard notion of isomorphism.

Moreover, we say that a partial function $f: D \rightarrow D^{\prime}$ is a partial homomorphism from $S$ to $S^{\prime}$ if for every $x$ and every $y$ in $D, R_{1} x y \rightarrow R_{1}^{\prime} f(x) f(y)$ and $R_{2} x y \rightarrow R_{2}^{\prime} f(x) f(y)$. Again, if $R_{3}$ and $R_{3}^{\prime}$ are empty, we obtain the standard notion of homomorphism as a particular case.

Note two crucial differences between partial isomorphism and partial homomorphism. First, a partial homomorphism does not require that the domains $D$ and $D^{\prime}$ of the partial structures under study have the same cardinality. Second, a partial homomorphism does not map the relation $R_{i}^{\prime}$ into a corresponding relation $R_{i}$ (it is a one-way mapping only). Clearly a partial homomorphism establishes a much less strict relation between partial structures.

Moreover, partial isomorphism and partial homomorphism offer mappings among partial structures that are less tight than their corresponding full counterparts-isomorphism and homomorphism. Partial mappings, as transformations that connect different partial structures that can be used in scientific practice, allow for the transferring of information from one domain into another-even when the information in question is incomplete.

We can then use partial structures and partial mappings to represent the models of the reducing and the reduced theories. This offers an additional source of openness for Fodor's account of reduction. The partial mappings make possible to 
connect different scientific domains with no requirement that they be structurally the same, since only some structure (the relations that are known to hold in the relevant domains) is transferred from one domain to another. And depending on the type of mapping that is used, different aspects of the partial structures in question are preserved. For instance, with a partial isomorphism, we are able to preserve in full certain parts of the structures under study, as long as the structures under consideration have the same cardinality. But clearly the last condition cannot always be met. In this case, a partial homomorphism between the structures will be more appropriate. In none of these cases, however, is it required that the objects in question have well-defined identity conditions, given that a formulation of partial structures can be given in a set theory that does not require that every object has well defined identity conditions (see Bueno 2000). ${ }^{8}$

The combination of unsharp mappings and partial mappings also allows us to accommodate the difficulties that plagued Nagel's proposal. (a) Clearly, there is no requirement of logical derivability of the special sciences from the reducing science. Instead, unsharp mappings and partial mappings are established between the relevant models. (b) It is perfectly viable to incorporate corrected versions of reduced theories by establishing new unsharp mappings and partial mappings between old and new theories. (c) Similarly to what goes on in Fodor's approach the multiple realizability phenomenon is accommodated with the introduction of disjunctive predicates, which, in turn, are related in the models of the reducing science via suitable unsharp mappings and partial mappings.

Note that this partial structure approach to "reduction", similarly to Fodor's, does not endorse reductionism. There is no place for natural kinds in it, nor is there any commitment to fundamental laws of nature. The autonomy of the special sciences is preserved throughout. However, the proposed framework offers a certain conception of the relation between the various sciences, keeping along the way the inevitable pluralism among the different domains of scientific inquiry. It seems to me that weaker forms of "reduction", such as the one suggested here, may play a role in contemporary scientific research. Anything significantly stronger seems to fly on the face of actual scientific activity.

\section{Conclusion}

If the considerations above are near the mark, just as with Fodor's own account, the use of unsharp mappings and partial mappings provides us with a minimal, weak conception of "reduction" without reductionism. And, by invoking these mappings, the partial structure proposal just sketched has the added bonus of being a little closer to scientific practice.

Principia 13(3): 269-82 (2009). 


\section{References}

Batterman, R. 2002. The Devil in the Details: Asymptotic Reasoning in Explanation, Reduction, and Emergence. New York: Oxford University Press.

Bueno, O. 2000. Quasi-Truth in Quasi-Set Theory. Synthese 125: 33-53.

- 2006. Representation at the Nanoscale. Philosophy of Science 73: 617-28.

Bueno, O.; French, S.; Ladyman, J. 2002. On Representing the Relationship between the Mathematical and the Empirical. Philosophy of Science 69: 497-518.

Carnap, R. 1928/1967. The Logical Structure of the World and Pseudoproblems in Philosophy. (Translated by Rolf A. George.) Chicago: Open Court.

Cohen, R. S.; Hooker, C. A.; Michalos, A. C.; van Evra, J. W. (eds.) 1976. PSA 1974: Proceedings of the 1974 Biennial Meeting of the Philosophy of Science Association. Boston: D. Reidel Publishing Company.

da Costa, N. C. A. \& French, S. 2003. Science and Partial Truth. New York: Oxford University Press.

Feigl, H. \& Maxwell, G. (eds.) 1962. Minnesota Studies in the Philosophy of Science, Volume 3. Minneapolis: University of Minnesota Press.

Feyerabend, P. 1962. Explanation, Reduction, and Empiricism. In Feigl and Maxwell (eds.) 1962, pp. 28-97.

Fodor, J. 1974. Special Sciences (Or: The Disunity of Science as a Working Hypothesis). Synthese 28: 97-115.

Fodor, J. 1997. Special Sciences: Still Autonomous After All These Years. Philosophical Perspectives 11: 149-63.

Frege, G. 1893/1903. Grundgesetze der Arithmetik, 2 volumes. Jena: Pohle. (Reprinted by Georg Olms, Hildesheim, 1962.)

French, S. \& Krause, D. 2006. Identity in Physics. Oxford: Clarendon Press.

Murphy, C. \& Coffer, J. 2002. Quantum Dots: A Primer. Applied Spectroscopy 56: 16A-27A.

Nagel, E. 1961 The Structure of Science. London: Routledge and Kegan Paul.

Schaffner, K. 1976. Reductionism in Biology: Prospects and Problems. In Cohen et al. (eds.) 1976, pp. 613-32.

Schrödinger, E. 1952. Science and Humanism. Cambridge: Cambridge University Press.

Sklar, L. 1967. Types of Inter-Theoretic Reduction. British Journal for the Philosophy of Science 18, pp. 109-24.

van Fraassen, B. C. 1989. Laws and Symmetry. Oxford: Clarendon Press.

OTÁvio BuEno

Department of Philosophy

University of Miami

Coral Gables, FL 33124-4670, USA

otaviobueno@mac.com

Resumo. Neste artigo, examino três modelos de redução. O primeiro, e mais restritivo, foi desenvolvido por Ernest Nagel como parte do programa empirista lógico. O segundo, articulado por Jerry Fodor, embora sendo significativamente mais amplo, é incapaz de dar sentido a uma característica saliente da prática científica. O terceiro modelo, que é também

Principia 13(3): 269-82 (2009). 
o mais condescendente, é desenvolvido empregando-se a abordagem baseada em estruturas parciais proposta por Newton da Costa e Steven French. Argumento que este terceiro modelo preserva os benefícios da proposta de Fodor e é ainda capaz de acomodar aspectos relevantes da prática científica. Em particular, ele oferece uma concepção de redução sem reducionismo, e descreve a relação entre a teoria que é reduzida e a que reduz por meio de mapeamentos não precisos e estruturas parciais — mesmo na presença de informação incompleta.

Palavras-chave: Redução, modelos, ciências especiais, estruturas parciais, mapeamentos parciais.

\section{Notes}

${ }^{1}$ It is a pleasure to dedicate this paper to Professor Newton da Costa, on the occasion of his $80^{\text {th }}$ birthday. Since my first publication-a joint paper with Professor Newton (as we call him in Portuguese)—so much of my work has been shaped and nurtured by his insightful and illuminating conception of the foundations of science, by his generous mind, and his critical acumen. His friendship, continuous help, and the collaboration with him mean the world to me. This paper, which uses the partial structures approach, is of course no exception among all of those works that rely on his sophisticated conception. I like to think that he will enjoy the paper, given that he is definitely not a reductionist-even though I am sure he will not approve of my loose use of the concept of model! (I promise I will not do that again when I write my paper celebrating his $90^{\text {th }}$ birthday!)

${ }^{2}$ I follow here the very lucid discussion offered by Robert Batterman (see Batterman 2002: 61-76).

${ }^{3}$ For a systematic and insightful discussion of this issue, see French and Krause 2006.

${ }^{4}$ According to this model:

$$
r=\epsilon h^{2} / \pi m_{t} e^{2}
$$

where $r$ is the radius of the sphere defined by the three-dimensional separation of the electron-hole pair; $\epsilon$ is the dielectric constant of the semiconductor; $m_{t}$ is the reduced mass of the electron-hole pair; $h$ is Planck's constant, and $e$ is the charge of the electron (see Murphy and Coffer 2002: 17A).

${ }^{5}$ I follow here da Costa and French [2003].

${ }^{6}$ For simplicity, I will take the partial relations in the definitions that follow to be two-place relations. The definitions, of course, hold for any $n$-place relations.

${ }^{7}$ A partial function is a function that is not defined for every object in its domain.

8 The set theory in question is quasi-set theory; for a thorough discussion, see French and Krause 2006.

Principia 13(3): 269-82 (2009). 